\title{
Thermodynamic analysis of the gas parameters distribution under leakage from a closed volume with rigid walls
}

\author{
Serhii Ponomarenko ${ }^{1,{ }^{*}}$, Oleksandr Zhevzhyk $^{2}$, Alex Vuginshteyn ${ }^{3}$, and Oleksandr Lutai ${ }^{4}$ \\ ${ }^{1}$ Institute of Geotechnical Mechanics named by N. Poljakov of National Academy of Sciences of \\ Ukraine, 49005, Dnipro, Simferopolska Str., 2a, Ukraine \\ ${ }^{2}$ Dnipro National University of Railway Transport named after Academician V. Lazaryan, 49010, \\ Dnipro, Lazaryana Str., 2, Ukraine \\ ${ }^{3}$ CICRAUN Internationally, 10005, New York, Wall Str., 30, USA \\ ${ }^{4}$ Institute of Strategic Technologies named after academician S.M. Koniukhov, 49005, Dnipro, \\ Simferopolska Str., 15, Ukraine
}

\begin{abstract}
The work deals with the thermodynamic analysis of the parameters of the gas in a closed volume with rigid walls, during its leakage and heat exchange with the environment. Based on the first law of thermodynamics, the law of Fick and the equation of the gas state, we obtained an equation that characterizes the effect of temperature gradient on the mass gas leakage in parallel processes of leakage and heat transfer. The regularity of the leakage coefficient change, determined by the diffusion coefficient, depending on the mass-average temperature in a closed thermodynamic system, is established. The variation of the leakage coefficient for the corresponding ranges of temperature gradients are shown. The research results can be implemented in the development of techniques and technologies for monitoring the tightness of hollow products under excessive pressure of the gas medium.
\end{abstract}

\section{Introduction}

The issues related to the loss of tightness of products and systems are particularly relevant due to the fact that the loss of tightness in some cases is not only unacceptable from a technical point of view, but also dangerous for staff and production in general. The loss of tightness can cause significant environmental damage; may have disastrous economic consequences. Currently, the problem of ensuring the tightness of products working under excessive pressure is becoming increasingly important for various industries, especially when detecting mini leaks on large volumes; in case of inaccessibility of the outer surface of sealed containers (closed with thermal insulation of the surface of the fuel tanks of launch vehicles, tanks dug into the ground and intended for the storage of petroleum products, compressed natural gas, toxic materials). Currently, the problem of ensuring the leakproofness of products working under excessive pressure is becoming increasingly

*Corresponding author: ovpt106@gmail.com 
important for various industries, especially when detecting mini leaks on large volumes; in case of inaccessibility of the outer surface of sealed containers (surfaces of the fuel tanks of launch vehicles covered with thermal insulation, tanks for the storage of petroleum products, compressed natural gas, toxic materials dug into the ground).

The most attractive in terms of industrial applications is a manometric method of tightness control, where the pressure drop of the test medium is recorded. These methods are classified as "pressure drop" methods. The main disadvantages of this method of estimating the gas pressure drop in the control unit per unit of time are the low value of the allowable decrease in gas pressure during the exposure time and the difficulty of determining its average temperature under very high requirements for its measurement accuracy $[1-6]$.

In classical thermodynamics, which studies the most common properties of macroscopic systems and methods of energy transfer and energy transformation, the issues of heat exchange with the environment are studied in detail, but the effect of heat transfer on the leakage of gaseous media has not been studied enough [7-11]. In this section of the phenomenological science, based on a generalization of experimental facts, the focus is on temperature gradients, and systems consisting of a very large number of particles are examined. In this regard, the methods of classical mechanics are not always acceptable to describe the behavior of the gaseous medium in two parallel processes - leakage and heat transfer, so, it is necessary to develop new thermodynamic descriptions for these processes.

It should also be noted that within the applied gas dynamics, a lot of attention was paid to the issues of gas leakage through various nozzles, but the problems of leaky vessels with microleaks of gas under conditions of heat exchange with the environment were not investigated [12 - 15].

The purpose of this work is to establish the thermodynamic laws of the processes of gas leakage from a closed volume with rigid walls and its heat exchange with the environment

\section{Methods}

We used the method of thermodynamic analysis of a leaky product with rigid walls, under the heat exchange with the environment; in this product the parameters of the gaseous medium change under the action of two parallel processes: leakage and heat transfer.

In the general case, the differential equation of energy balance for any thermodynamic system is $[8-11,16]$ :

$$
d Q^{t}=d K+d P+d L
$$

where $d Q^{t}$ is some heat supplied to the system (or removed); $d K$ is the change in the kinetic energy of gas molecules due to the heat supplied (removed); $d P$ is a change in potential energy of gas molecules; $d L$ is an external work done by gas molecules.

To calculate the amount of heat supplied (reduced) $Q^{t}$ per unit of time $\tau$, formula of Newton $[11,16]$ is usually used, in which the heat flux is considered proportional to the temperature difference between the heat carrier and the wall:

$$
\frac{Q^{t}}{\tau}=\alpha\left(T_{t}-T_{f}\right) F,
$$

where $\alpha$ is a heat transfer coefficient, $\mathrm{W} /\left(\mathrm{m}^{2} \cdot \mathrm{K}\right) ; T_{t}$ is a heat carrier temperature, $\mathrm{K} ; T_{f}$ is a wall temperature, $\mathrm{K} ; F$ is an area of surface of contact between the heat carrier and the wall, $\mathrm{m}^{2}$.

It should be noted that the Newton's formula is a formal expression of the heat flux and 
does not explicitly take into account all factors affecting the heat transfer intensity $[11,16]$. Therefore, this effect should be taken into account by the heat transfer coefficient.

In practice, the intensity of heat transfer is usually unequal over the entire area of contact of the heat carrier with the wall and the heat transfer coefficient has different values for different parts of the contact surface.

In the general case, the change in the internal energy of the gas $d U$ is equal to $d U=d K+d P$, and the elementary deformation work (external work) of gas in volume $V$ under pressure $P$ is equal to $d L=P d V[8-11,16]$. Given that a closed volume with rigid walls is considered, in our case the external work will is zero and equation (1) for one kilogram of gas can be rewritten as:

$$
d Q^{t}=d U
$$

so, heat supplied or removed is spent only on changing the internal energy of the gas and $Q^{t}=\Delta U$.

For an ideal gas with a constant heat capacity $c_{V}$, the change in internal energy, taken into account two parallel processes, heat transfer and leakage, is equal to:

$$
\Delta U=c_{V}\left(m_{2} T_{2}-m_{1} T_{1}\right),
$$

where $m_{2}$ and $m_{1}-$ are final and initial mass of gas, $\mathrm{kg} ; T_{2}$ and $T_{1}-$ final and initial gas temperature, $\mathrm{K}$.

It should be noted that the temperature of the gas medium in the volume may have different values. In general, the expression for the average temperature $T_{s, i}(i=1 ; 2)$ of the gaseous medium in a closed volume is:

$$
T_{s, i}(\tau)=\frac{1}{m_{i}} \iiint \rho\left(x_{i}, y_{i}, z_{i}, \tau\right) \cdot T\left(x_{i}, y_{i}, z_{i}, \tau\right) \cdot d x_{i} d y_{i} d z_{i} \quad(i=1 ; 2),
$$

where $m_{i}$ is a mass of gas in a closed volume, $\mathrm{kg} ; \rho\left(x_{i}, y_{i}, z_{i}, \tau\right)$ is a density of gas, $\mathrm{kg} / \mathrm{m}^{3} ; T\left(x_{i}, y_{i}, z_{i}, \tau\right)$ is thermal field distribution function, $\mathrm{K} ; x_{i}, y_{i}, z_{i}$ are corresponding coordinates, $\mathrm{m}$.

This equality shows that the average temperature of the gas in a closed volume is the mass-average temperature of the gas medium. Consequently, the temperatures in equality (4) $T_{2}$ and $T_{1}$ are mass-average values.

Taken into account (2) and (4) and the fact, that $m_{2}=m_{1}-m_{f}\left(m_{f}-\right.$ mass of gas leaked due to leakage of the product, $\mathrm{kg}$ ) equation (3) takes the form:

$$
\alpha\left(T_{t}-T_{f}\right) F \tau=c_{V}\left(m_{2} T_{2}-m_{1} T_{1}\right)=c_{V}\left\lfloor m_{1}\left(T_{2}-T_{1}\right)-m_{f} T_{2}\right\rfloor
$$

In the problems of gas outflow from a product, where the pressure is greater than the surrounding pressure, two cases are possible: when the flow rate is less than the critical one and when the flow rate is equal to the critical speed [12 - 15]. However, the magnitude of the gas leakage depends on the location, configuration, and size of the orifice area, which are unknown. Therefore, the use of the theory of outflow from a hole in a product with pressure is not possible in this case.

Without study of the localized process of gas outflow, we consider the movement of gas through the walls of the product, averaging this process over the entire surface of the product. Since the gas consumption in this problem is very small, we interpret its movement through the product wall as diffusion and use Fick's law $[5,10]$ : 


$$
\frac{d m}{d \tau}=-D \frac{\Delta \rho}{\Delta x} S
$$

here $m$ is mass of gas transferred by diffusion, $\mathrm{kg} ; D$ is effective coefficient of gas diffusion through the product wall, $\mathrm{m}^{2} / \mathrm{s} ; \Delta \rho / \Delta x$ is a density gradient of a substance along the coordinate in the direction of mass transfer, $\mathrm{kg} / \mathrm{m}^{4} ; S$ is mass transfer surface area, $\mathrm{m}^{2}$.

In this task $\Delta \rho=\frac{m_{2}-m_{1}}{V}=-\frac{m_{f}}{V}, \mathrm{~kg} / \mathrm{m}^{3} ; \Delta x=\delta$ is a wall thickness, $\mathrm{m}$. Considering this and integrating equality (6), we have:

$$
\frac{1}{\tau}=D \frac{S}{\delta V}
$$

whence it follows that the time of leakage of gas from the product will be equal to:

$$
\tau=\frac{\delta V}{D S}
$$

Thus, the time during which $m_{f}$ of gas flows out of the product due to its leakage is directly proportional to the wall thickness and free volume of the product, inversely proportional to the diffusion coefficient and the surface area of the product. In this case, the diffusion coefficient is a quantitative characteristic of the diffusion rate, which depends on temperature and is determined by the properties of the medium, as well as the type of diffusing particles.

According to the molecular-kinetic theory, the diffusion coefficient characterizes the mass transfer rate and is determined by the formula $D=\frac{1}{3}\langle u\rangle\langle l\rangle$, where $\langle u\rangle$ is average arithmetic speed of molecules, $\mathrm{m} / \mathrm{s} ;\langle l\rangle$-is average free path of molecules, $\mathrm{m}$. As $\langle u\rangle \sim \sqrt{T}$, and $\langle l\rangle \sim 1 / P \sim 1 / T$, wheree $P$ and $T$ are respectively, the pressure and temperature of the gas, the diffusion coefficient is proportional to $D \sim \sqrt{T} / P \sim 1 / \sqrt{T}$.

Taking into account equality (7), equation (5) takes the form:

$$
\alpha\left(T_{t}-T_{f}\right) \frac{\delta V F}{D S}=c_{V}\left[m_{1}\left(T_{2}-T_{1}\right)-m_{f} T_{2}\right]
$$

After solving relatively $m_{f}$, we have:

$$
m_{f}=m_{1} \frac{T_{2}-T_{1}}{T_{2}}-\frac{\alpha \delta V F}{c_{V} D S} \frac{T_{t}-T_{f}}{T_{2}}=m_{1}\left(1-\frac{T_{1}}{T_{2}}\right)-\frac{\alpha \delta V F}{c_{V} D S} \frac{T_{t}-T_{f}}{T_{2}} .
$$

or, using the equation of state for an ideal gas:

$$
m_{f}=\frac{P_{1} V}{R T_{1}}\left(1-\frac{T_{1}}{T_{2}}\right)-\frac{\alpha \delta V F}{c_{V} D S} \frac{T_{t}-T_{f}}{T_{2}},
$$

where $P_{1}$ is the initial value of the gas pressure in the volume, $\mathrm{Pa} ; R$ is universal gas constant, $\mathrm{J} /(\mathrm{kg} \cdot \mathrm{K})$.

The first member of the right side of equation (8) is the change in mass of a gas in a closed volume with rigid walls under pressure, and the second is the change in mass of this 
gas due to heat exchange with the environment and gas leakage (diffusion) into the environment.

The heat capacity of an ideal gas does not depend on temperature and, using well known in thermodynamics ratio $c_{V}=R /(\gamma-1)$, where $\gamma$ is an adiabatic index (Poisson's ratio), equation (8) is converted to:

$$
m_{f}=\frac{V}{R}\left[\frac{P_{1}}{T_{1}}\left(1-\frac{T_{1}}{T_{2}}\right)-\frac{\alpha \delta F}{D S}\left(\frac{T_{t}}{T_{2}}-\frac{T_{f}}{T_{2}}\right)(\gamma-1)\right]
$$

Equation (9), obtained on the basis of the first law of thermodynamics, Fick's law and the gas state equation, characterizes the influence of gas-dynamic parameters (temperature and pressure) on the mass of gas that has flowed out from a closed volume with rigid walls due to its leakage. Taking into account that it is difficult to specify some parameters in this equation ( $\alpha, \delta, F, S$ and $\left.T_{f}\right)$, it is very problematic to apply equation (9) to quantify the mass of gas leakage. However, this equality can be applied for a qualitative assessment of the influence of heat exchange processes and leakage on the value of mass of gas leakage in the product.

Since the process of gas leakage through existing leakages in the joints or microcracks of the product walls has a very low flow rates at the molecular level, it is quite legitimate to consider it as the equivalent of some diffusion described by Fick's law and to determine according to the formula (6). However, when the product has a complex shape and configuration, it is rather difficult to determine the equivalent wall thickness of the product $\delta$, the total surface area $F$ and the leakage surface area $S$. For this case, the entire leakage ratio $K_{S}$ can be identified as

$$
K_{S}=\frac{\alpha \delta F}{D S}
$$

Taking into account that $\alpha, \delta, F$ and $S$ are constant values, it can be stated that $K_{S} \sim 1 / D$.

Considering the wall temperature of the product as a certain mean value $T_{f}=\left(T_{t}+T_{1}+T_{2}\right) / 3$, taken into account (10), equation (9) is converted to:

$$
m_{f}=\frac{V}{R}\left[\frac{P_{1}}{T_{1}}\left(1-\frac{T_{1}}{T_{2}}\right)-\frac{K_{S}}{3}\left(\frac{T_{t}-T_{1}}{T_{2}}-1\right)(\gamma-1)\right] .
$$

To estimate an assessment of the heat transfer effect to mass gas leakage, we consider in the inequality obtained from equality (11) taking into account the fact that, in its physical sense, $m_{f}>0$ :

$$
\left(1-\frac{T_{1}}{T_{2}}\right)>\frac{T_{1} K_{S}(\gamma-1)}{3 P_{1}}\left(\frac{T_{t}-T_{1}}{T_{2}}-1\right)
$$

At ambient temperature $T_{t} \leq T_{1}$, the right side of inequality (12) is always negative, and its left side can be as positive $\left(T_{1} \leq T_{2}\right.$ despite the leakage, inside the considered volume of the product, due to its heat exchange with the environment, gas is heated, which causes a slight increase in gas pressure), as negative $\left(T_{1}>T_{2}\right.$ gas leakage and heat exchange of the product with the environment cause a decrease in the temperature and pressure of the gas in the product). At the same time, while the gas temperature in the product decreases, the leakage coefficient $K_{S}$ decreases due to an increase in the diffusion coefficient (taking into 
account, as shown above, its inversely proportional dependence on temperature), and an increase in the gas temperature leads to an increase of $K_{S}$.

When $T_{t}>T_{1}$, and $\left(T_{t}-T_{1}\right) / T_{2}<1$, (this is possible in the case when the difference between average mass temperatures $T_{t}-T_{1}$ is less than the average mass temperature $T_{2}$ ) the picture of the processes of leakage and heat exchange is similar to the above described case of the negative value of the right side of inequality (12).

While $T_{t}>T_{1}$ and $\left(T_{t}-T_{1}\right) / T_{2} \geq 1$ (the average mass temperature $T_{2}$ does not exceed the difference between the average mass temperatures $T_{t}-T_{1}$ ), the right side of inequality (12) is positive. This means that inside the volume, due to the heat exchange of the product with the environment, there is a significant heating of the gas with an increasing its pressure $T_{2} \gg T_{1}$ and the left side of inequality (12) has a positive value, and due to a decrease in the diffusion coefficient under a general increase in gas pressure, the leakage coefficient $K_{S}$ is increased within the limits of a change in the mean-mass gas temperature in the product.

For this case, taking $T_{1} / T_{2} \rightarrow 0$ and $1 / T_{2} \approx 0$, inequality (12) can be written in the form:

$$
0<K_{S}<\frac{3 P_{1} T_{2}}{T_{1} T_{t}(\gamma-1)},
$$

and equation can be written (11) as:

$$
m_{f}=\frac{V}{R}\left[\frac{P_{1}}{T_{1}}-\frac{K_{S}}{3} \frac{T_{t}}{T_{2}}(\gamma-1)\right] .
$$

And finally, when $T_{t} \approx T_{1} \approx T_{2}=T$ (the temperature field inside the volume is uniform, stationary and in thermal equilibrium with the environment), inequality (12) is satisfied regardless of the gas temperature; the value of leakage, in accordance with equation (11), is determined only by its properties and diffusion characteristics (the degree of leakage of the product):

$$
m_{f}=\frac{V K_{S}}{3 R}(\gamma-1)
$$

\section{Results and discussion}

Thermodynamic analysis of equations (11), (14) and (15) shows that a change of the temperature field of a gas inside a closed volume of a product with rigid walls, caused by a leak and heat exchange with the environment, does not have a significant effect on the value of gas leakage from this volume. At the same time, the influence of temperature gradients (increase or decrease in $T_{2}$ ) to the mass gas leakage is compensated by a corresponding change in the leakage coefficient (increase or decrease of $K_{S}$ ) due to a change in the diffusion coefficient.

\section{Conclusions}

The defining of thermodynamic laws of joint processes of gas leakage from a closed volume with rigid walls and its heat exchange with the environment is an important scientific and technical issue, that solves the problems of developing technology and techniques for determining the degree of leakage of hollow products under excessive pressure of a gas medium. The justification of compensating the effect of temperature gradients on the mass gas leakage by changing the diffusion coefficient and, as a result, by 
changing the leakage coefficient, allows us to broaden our understanding of the joint parallel processes of leakage and heat transfer. As the results of research we can formulate new directions in the field of non-destructive tightness control of products and their systems.

\section{References}

1. Zazhigin, A.S. (1975). Sredstva kontrolya germetichnosti. Moskva: Mashinostroyeniye

2. Levina, L.E., Sazhin, S.G. (1980). Manometric method of leak testing. Russian Journal of Nondestructive Testing, 11, 45-51

3. Sazhin, S.G. (2009). Analysis of modern methods and means of the control of tightness of technological objects. V mire NK, 2 (44), 4-7

4. Bulat, A., Voloshyn, O., Ponomarenko, S., Gubenko, D. (2013). New-generation technique and technology for leakage tests. VII International scientific-practical conference "School Underground Mining". Annual Scientific-Technical Collection "Mining of Mineral Deposits". Netherlands: CRC Press / Balkema. 1-4

5. Voloshyn, A.I., Bulat, A.F., Ponomarenko, S.N., Gubenko, D.I., Kutumov, I.V. (2016). Precision means for checking the tightness. Mariupol: Skhidnyy vydavnychyy dim

6. Voloshyn, A.I., Ponomarenko, S.N. Gubenko, D.I. (2014). Leakage monitoring of compressed air mine pipe-lines according to the fixed volumes method, Geotehnicheskaya mehanika, 119, 42-52

7. Loitsyansky, L.G. (2003). Mekhanika zhidkosti i gaza. Moskva: Drofa

8. C. Borgnakke, R.E. Sonntag, Fundamentals of thermodynamics (Hoboken, Wiley, 2009)

9. H.A. Buchdahl, The Concepts of Classical Thermodynamics (Cambridge, Cambridge University Press, 2009)

10. Kuhling, H. (1985). Spravochnik po fizike. Moskva: Mir

11. Dryzhakov, E.V., Kozlov, N.P., Korneichuk, N.K. (1971). Tekhnicheskaya termodinamika. Moskva: Vysshaya shkola

12. Abramovich, G.N. (1969). Prikladnaya gazovaya dinamika. Moskva: Nauka

13. E. Rathakrishnan, Gas dynamics (Delhi, PHI Learning Pvt. Ltd., 2013)

14. Carabin, A.I. (1964), Szhatyy vozdukh: vyrabotka, potrebleniye, puti ekonomi. Moskva: Mashinostroyeniye

15. Sokolov, E.Ya., Singer, N.M. (1989). Struynye apparaty. Moskva: Energoatomizdat

16. Voloshyn, A.I., Ponomarenko, S.N. Gubenko, D.I. (2015). The equation of thermal energy balance for the leak control by method of fixed volumes. Geotehnicheskaya mehanika: sbornik nauchnykh trudov, 122, 26-37 